Article

\title{
High-Precision Solvent Vapor Annealing for Block Copolymer Thin Films
}

\author{
Gunnar Nelson, Chloe S. Drapes, Meagan A. Grant, Ryan Gnabasik, Jeffrey Wong \\ and Andrew Baruth* \\ Department of Physics, College of Arts and Sciences, Creighton University, 2500 California Plaza, \\ Omaha, NE 68178, USA; GunnarNelson@Creighton.edu (G.N.); ChloeDrapes@Creighton.edu (C.S.D.); \\ MeaganGrant@Creighton.edu (M.A.G.); gnaba001@umn.edu (R.G.); JeffreyWong@Creighton.edu (J.W.) \\ * Correspondence: AndrewBaruth@Creighton.edu; Tel.: +1-402-280-2644
}

Received: 19 April 2018; Accepted: 25 May 2018; Published: 29 May 2018

\begin{abstract}
Despite its efficacy in producing well-ordered, periodic nanostructures, the intricate role multiple parameters play in solvent vapor annealing has not been fully established. In solvent vapor annealing a thin polymer film is exposed to a vapor of solvent(s) thus forming a swollen and mobile layer to direct the self-assembly process at the nanoscale. Recent developments in both theory and experiments have directly identified critical parameters that govern this process, but controlling them in any systematic way has proven non-trivial. These identified parameters include vapor pressure, solvent concentration in the film, and the solvent evaporation rate. To explore their role, a purpose-built solvent vapor annealing chamber was designed and constructed. The all-metal chamber is designed to be inert to solvent exposure. Computer-controlled, pneumatically actuated valves allow for precision timing in the introduction and withdrawal of solvent vapor from the film. The mass flow controller-regulated inlet, chamber pressure gauges, in situ spectral reflectance-based thickness monitoring, and low flow micrometer relief valve give real-time monitoring and control during the annealing and evaporation phases with unprecedented precision and accuracy. The reliable and repeatable alignment of polylactide cylinders formed from polystyrene- $b$-polylactide, where cylinders stand perpendicular to the substrate and span the thickness of the film, provides one illustrative example.
\end{abstract}

Keywords: block polymers; self-assembly; thin films; solvent vapor annealing; nanolithography

\section{Introduction}

Techniques to achieve periodic nanostructures via traditional "top down" methods, including photolithography, have become increasingly challenging within the semiconductor industry. Ultra-small feature production is approaching fundamental resolution limits $(193 \mathrm{~nm}$ ultraviolet (UV) lithography, for example, recently reaching sub-30 nm features) [1-11]. One promising strategy is investigating "bottom up" approaches that rely on nanoscale self-assembly. In 2007, directed self-assembly was first considered as a potential scaling solution, according to the International Technology Roadmap for Semiconductors (ITRS) [11]. In their 2013 report, the directed self-assembly of complex structures with low anneal time, low defect density, and high reproducibility was identified as one of the "Grand Challenges" to extend Moore's law [11]. The directed self-assembly of block polymer (BP) thin films has become a particularly strong candidate to achieve sub- $20 \mathrm{~nm}$ dimensions, where the size and morphology is controlled by varying the molecular weight of the constituent polymer blocks.

Due to thorough investigations over decades, the BP research community generically considers the bulk behavior of many BPs well known $[12,13]$. Bulk morphologies are characterized by the 
Flory-Huggins interaction parameter $(\chi)$, the degree of polymerization $(\mathrm{N})$, the volume fractions of the constituent blocks (f), and the particular architecture [13]. Furthermore, much is known about bulk behavior under a variety of stimuli, whether thermal, solvent, or mechanical [12]. In particular, measured or known quantities can well predict the alignment of structures and morphologies within the bulk. On the contrary, the confinement of a thin film and the associated surface energy contributions introduces additional confounders [14,15]. In particular, the complexity of surface interactions (both with the substrate and free surface) impose new, often asymmetric, boundary conditions [16,17]. Regardless, techniques designed to promote ordering of BP thin films have progressed rapidly, driven by applications requiring long-range lateral ordering, uniformity in feature size and high placement precision. Most of the historical approaches to BP ordering have been largely unpredictable and are often slow or energy-intensive: including thermal annealing [18,19], electric field alignment [20,21], and incorporation of low surface energy midblocks [22,23]. More recently, the use of pre-patterned substrates (chemical or topographical) to act as a guide for BP assembly has been successfully incorporated but can be time-intensive and involves multiple lithographic approaches [24]. Therefore, it is not necessarily cost-effective for high-throughput applications. As a result, a maturing technique is solvent vapor annealing (SVA) [25].

SVA was originally introduced as an alternative to thermal annealing for BPs exhibiting thermal degradation, problematic thermally-driven transitions, or slow dynamics due to high molar mass [26-28]. The interest in SVA of BPs has grown well beyond this in recent years. It has been shown to optimize organization quickly due to the increased chain mobility, a possibly decreased $\chi$ (dependent on solvent polarity), and tunable surface energies [25]. In this process, a BP film is exposed to the vapors of one or more organic solvents, offering direct control over lyotropic transitions (cylinders to spheres, for example) while in the solvated state as well as during evaporation [29,30]. This technique also has the ability to reduce defect density dramatically [31], while improving lateral ordering, both at the free surface and into the bulk of a film. This ordering is achieved more quickly (by several orders of magnitude) and completely than previous methods [32]. Although much is known about the interactions of BPs with solvent in bulk [33], the effects in thin films exhibit different behaviors due to the presence of confining surfaces and the dynamic exchange with the solvent vapor atmosphere [17]. Thus, this technique continues to suffer from reliability problems and no standardized methods have become apparent. It is becoming increasingly clear that a continued understanding of the specific ordering mechanisms of a BP system is paramount, where the quest for generic understanding of any BP thin film system remains elusive.

Of critical importance to many technological applications is the ability to direct BP thin films to form cylinders that stand perpendicular to the surface, traverse through the thickness of the film, and laterally pack with hexagonal order. A recently demonstrated approach to nanolithography of a magnetic thin film, for example, utilized such BP-based lithography masks using a Damascene-like approach. This approach was able to synthesize hexagonally-packed magnetic nanodots with a diameter of $19 \mathrm{~nm}$ with high fidelity and retention of robust ferromagnetism [34]. Furthermore, this approach achieved diameter control, down to $14 \mathrm{~nm}$, and the potential for high-temperature processing with an additional atomic layer deposition step of $\mathrm{ZnO}$ [35]. These techniques rely on the vertical alignment of cylinders. On the contrary, many current advances in BP alignment, including important work in solvent concentration gradients [36], have focused solely on the free surface of the film. This is insufficient to be a direct substitution for most traditional lithographic approaches [37]. Recent results reveal that optimized ordering of hexagonally-packed cylinders can potentially occur in seconds and extend through the film using SVA, but this approach can be somewhat unreliable [38], where near $100 \%$ success of forming the desired morphology has yet to be achieved. Advances in computer simulation as well as in situ X-ray and neutron scattering continue to both further understand this process and improve upon it [39-41]. While such an understanding of the self-assembly behavior is critical to the advancement of this field, utilizing a statistical approach to quantifying SVA with large sample sets, with a goal of overcoming reliability barriers, requires the ability to identify, measure 
and develop controls for all of the pertinent variables with reliable precision. This includes chamber pressure, solvent exposure time, solvent concentration in the film, solvent evaporation rate, solvent purity or combinations, ambient temperature, sample and solvent temperature, humidity [42], and film thickness to name a few. These are addressed in the present manuscript, with a primary focus on the role of controlling chamber pressure and solvent evaporation rates at fast time scales of $\sim 10$ ms. We note that this chamber does not actively control sample [41] or solvent temperature [43-45], or include multiple solvents [46], which have been shown important to controlling annealing kinetics. So, these results work to keep these parameters as consistent as possible. To that end, we present here our strict annealing protocols and our climate-controlled SVA chamber with computer-controlled solvent vapor flow and pressure management and in situ spectral reflectance-based solvent concentration $(\phi)$ measurements. This chamber allows us to fix potential variables while investigating only one. This leads to unprecedented control over the SVA process with a goal of systematic studies with high reliability and repeatability that may have advantages as BP SVA alignment moves from its current research phase (relying heavily on in situ X-ray and neutron scattering) into scaled-up processing. One critical and unprecedented advancement is the ability to stabilize solvent concentration within a BP thin film for an arbitrarily long time at a user-defined chamber pressure, a necessary prerequisite for any temporal studies of crystallization.

To date, there are three primary methods of controlled SVA in the literature with differing levels of complexity, as illustrated in Figure 1. These processes were recently reviewed by Posselt et al. and Gu et al. [32,47], where each method typically incorporates an optical interferometer to monitor thickness (i.e., swelling due to solvent uptake) in real time that is placed above the chamber and shines down on the sample surface. Briefly, in "jar annealing," depicted in Figure 1a, a solvent reservoir is placed in a sealed vessel with the BP film. The solvent vapor pressure, and thus the film swelling, is parameterized by the ratio of liquid solvent surface area to the chamber volume [48]. The solvent evaporation is either done via opening the lid, which is difficult to quantify, or introducing a leak until all solvent has evaporated $[48,49]$. A natural extension of this SVA method is the inclusion of inlet/outlet flow lines, as seen in Figure 1b, where the incorporation of an inert gas flow can serve to modify the vapor pressure and BP film swelling more directly [50]. However, there is still only limited control over the solvent evaporation rate. The next extension is displacing the solvent reservoir from inside the sealed chamber into a separate sealed reservoir, as shown in Figure 1c. In this SVA method, solvent is carried in the vapor phase by a carrier gas into the chamber. Both the absolute pressure and vapor pressure inside the chamber are controlled via the flowrates of the vapor line and a second inlet for an inert gas stream. This gives significant dynamic control of BP film swelling, although the highest achievable vapor pressure is reduced compared to Figure $1 \mathrm{a}, \mathrm{b}$ due to a dilution of vapor from the carrier gas. The solvent evaporation rate is controlled in a similar fashion to the method depicted in Figure 1b, but without the presence of the solvent reservoir, thus offering more control. Similar methods have proliferated [30,51-53], but only few utilize real-time computer control $[41,45]$ and there is currently an inability to maintain a fixed solvent concentration for an arbitrarily long time scale. The present manuscript describes a new evolution in the SVA method that builds on these existing models, adding additional control over solvent introduction into and evaporation out of a BP film with a high degree of reliability and reproducibility by controlling the vapor pressure through inlet/outlet flows.

Several recent in situ studies utilizing Grazing Incidence Small Angle X-ray and Neutron Scattering (GISAXS and GISANS) serve to motivate the ability to stabilize a BP film with a specific solvent concentration $[41,54]$. These studies have shown to be critical in identifying the role of the order-to-disorder transition in directed self-assembly, as well as the role of solvent polarity and temperature. Their results could be further extended with an increased level of control over solvent concentration during the anneal and during the solvent evaporation. For example, Gu et al. show a high degree of ordering in poly(styrene)- $b$-poly(2-vinyl pyridine) using GISAXS with an increasing solvent concentration [30]. Upon rapid evaporation, scanning electron micrographs (SEM) of the resultant films exhibit long correlation lengths of in-plane cylinders. Furthermore, 
recent results indicate that approaching a solvent concentration consistent with the order-to-disorder transition leads to the largest correlation lengths [32]. This solvent concentration is also consistent with enhanced defect removal [31]. Annealing at solvent concentrations above the order-disorder transition will, instead, result in a potentially disordered film, as previously shown [34]. In addition, Sinturel et al. revealed similar ordering in a solvated poly(styrene)- $b$-poly(lactide) film, where GISAXS was monitored with increasing solvent concentration [29]. The data shows that the correlation length of perpendicularly aligned cylinders in quickly dried films is largest for a high solvent concentration (below the order-to-disorder transition). Finally, Berezkin et al. recently showed how mixed solvents and the use of elevated temperature in a controlled SVA chamber (similar to Figure 1c) further enhance control over final morphologies. These GISAXS studies, while extremely insightful, rely on somewhat uncontrolled solvent exposure methods, as presented in Figure 1. In each case, no static solvent concentration (often described as swelling ratio) was achieved over any appreciable amount of time. Such control is a prerequisite for any temporal study of ordering kinetics and further optimization of an annealing protocol. Moving forward, especially when considering scaling up these techniques towards mass production, creating a highly controlled SVA chamber could assist in avoiding the use of continued in situ X-ray or neutron scattering techniques that are not easily extensible to scaling up. Instead, a highly controlled SVA chamber could establish reliability and repeatability that incorporates the critical parameters that have been established from these scattering techniques.

a)

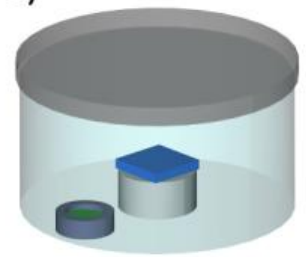

b)

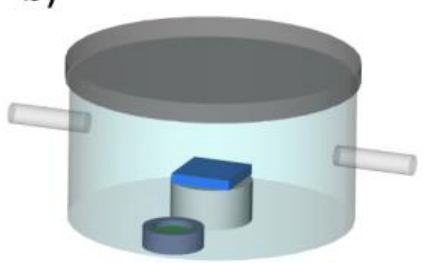

c)

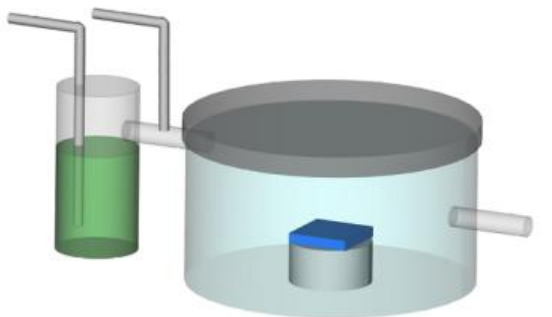

Figure 1. Primary methods of solvent vapor annealing; (a) "jar annealing," where a sealed chamber contains the sample and a solvent reservoir; (b) "jar annealing" with inlet/outlet lines for inert gas flow; (c) solvent vapor flow via a carrier gas through inlet/outlet lines. Block polymer films are depicted in blue, with liquid solvent depicted in green.

In addition to a controlled static solvent concentration, the evaporation rate serves as another complicating factor to be addressed. Solvent evaporation rate has been shown to strongly affect the surface morphology of BP films [52], where the evaporation has been shown to produce an ordering front that propagates through the film from the free surface to the substrate [55]. The timing of solvent removal dominates this effect. Enviable results were reported in 2004 from Kim et al. revealing defect-free ordering of cylinders at the free surface over large lateral length scales, where GISAXS data aimed to expose the role of this ordering front [55]. The ordering depends strongly on the exact trajectory through the BP phase diagram as solvent is removed [56], with changes in polymer dynamics and order-order transitions serving as potential complicating factors [57]. As such, conflicting results dictate that either slow [51,52] or fast [58] evaporation times may lead to ideal morphologies. Recently, studies of solvent concentration profiles [58] have suggested that the cylinder growth rate during evaporation is a product of the polymer chain mobility and the driving force to phase separate. Dynamical field theory simulations from Paradiso et al. further expose the role of rapid solvent evaporation and segregation strength $(\chi)$ on cylinder orientation [57]. It is increasingly clear that the ability to systematically remove solvent from the film, including at rapid time scales, is important to understanding final film morphologies. In particular, revealing how a given morphology propagates into the film during solvent evaporation, which is a necessary requirement for many nanolithographic applications. It is important to note that the role of solvent removal on rapid time scales (less than $100 \mathrm{~ms}$ ) has not been successfully monitored using GISAXS, where integration times 
can be somewhat long (1-10 s) [32]; however, recent use of charge-coupled device (CCD) cameras have been shown to be effective for studying fast kinetics [47]. This opens the door for quantifying the effects of solvent removal in real time. Presently, in many of these studies, combining known structural properties from GISAXS in the solvated state with final morphologies imaged with atomic force microscopy (AFM) or SEM following rapid evaporation, as addressed above, have been used to reveal important relationships between evaporation trajectories and final morphologies. The present manuscript describes a systematically improved SVA method that offers a potential to elucidate multiple key variables in the SVA process and offers a level of control, reliability and reproducibility to enhance the understanding of ordering kinetics both during annealing and, critically, evaporation. Specifically, this improved SVA method monitors and controls chamber pressure, solvent exposure time, solvent concentration in the film, solvent purity and solvent evaporation rate, while concurrently monitoring ambient temperature and humidity.

\section{Materials and Methods}

\subsection{Solvent Vapor Annealing}

Optically polished substrates were single-crystalline silicon $<111>$ wafers $(\mathrm{n}$-type, $5 \Omega \cdot \mathrm{cm}$ ) with a native oxide layer (University Wafer, South Boston, MA, USA). No attempt was taken to remove the native oxide. Toluene (ACS Certified), tetrahydrofuran (THF) (Certified, contains $0.025 \%$ butylated hydroxytoluene as a preservative), acetone (99.5\%), methanol (ACS Certified), and sodium hydroxide granules were all purchased from Fisher Chemical, Pittsburgh, PA, USA. 1,1,1,3,3,3-Hexamethyldisilazane (HMDS) (98\%) and $3 \AA$, 4 to 8 mesh, 3333 were purchased from Acros Organics, Belgium, WI, USA.

Construction of the solvent vapor-annealing chamber (SAC) required chemically inert stainless-steel tubing and valves with Swagelok tube fittings. Pneumatically actuated stainless steel ball valves (1/4" and 3/4") utilizing Dow Corning M111 (heavy-consistency dimethyl silicone compound) lubricant and Modified PTFE packing (SS-T12-S-065-20 and SS-45S8-33C), stainless steel quarter-turn plug valves $\left(1 / 4^{\prime \prime}\right)$ with Kalrez O-rings (SS-4P4T), a stainless steel low-flow metering valve (1/4") utilizing a Kalrez O-ring (SS-SS4-KZ-VH), a stainless steel low-pressure (5 psig/34.5 kPa) unfilled pressure gauge (LP1-SS-254-5PSI), a stainless steel unfilled pressure gauge (60 psig/413.7 kPa, PGI-63C-PG60-LAOX), and 1/4" and 3/4" stainless steel tubing (SS-T4-S-035-20 and SS-T12-S-065-20, respectively) were purchased from the Swagelok Company, Saarland, OH, USA. The body and bubbler portions of the SAC utilized vacuum-grade Conflat flanges with oxygen-free copper gaskets. Stainless steel tees (275-150-CFT), 6-way cube (275-CUBE-OS), and all Conflat blanks (275-000-T) and Swagelok adaptors were purchased from LDS Vacuum Products, Inc., Longwood, FL, USA, or modified in-house. Conflat flanged zero length deep UV quartz (Corning HPFS 7980 Fused Silica) viewport was purchased from the Kurt J. Lesker Company, Jefferson Hills, PA, USA (VPZL-275Q). Dry $\mathrm{N}_{2}$ inlet was achieved with a Drierite gas purifier, and was controlled via an Apex 500 SCCM mass flow controller with RS-232 digital control (Schoonover Inc., Canton, GA, USA). Dry $\mathrm{N}_{2}$ pressure from the gas tank were monitored and controlled with a dual-stage gas regulator $(0-344.7 \mathrm{kPa})$ and flowrates were monitored and controlled with a Panel-Mount Flowmeter (OMEGA Engineering, Stanford, CT, USA) for air, with a brass valve, with two different flow ranges (3-30 SCFH and 30-300 SCFH) from McMaster-Carr, Elmhurst, IL, USA. Pneumatic valve control was achieved with 3-way 1/4" NPT, normally closed, 120 V, 100 psi solenoids, various One-touch fittings (1/4") and 1/4" nylon tubing purchased from the Swagelok Company, Saarland, OH, USA. Electric control of pneumatics was achieved with a National Instruments USB, 8 input, 12-Bit, 10 kS/s, Multifunction DAQ; 25 A, 250 V solid state relays; and LabVIEW 2016 software (National Instruments, Austin, TX, USA). Finally, film thickness was determined in situ with spectral reflectance via a Filmetrics F20-UV, San Diego, CA, USA, general-purpose film thickness measurement system with both halogen and deuterium sources. 


\subsection{Synthesis of Poly(Styrene)-Block-Poly(Lactide)}

The synthesis of poly(styrene)-block-poly(lactide) (PS-b-PLA) is described fully elsewhere [34]. Succinctly, hydroxyl-terminated PS $(\mathrm{Mn}=42.5 \mathrm{kDa}$ ) was synthesized via living anionic polymerization. The subsequent PLA was synthesized via ring opening transesterification polymerization (ROTEP) of D,L-lactide in dichloromethane at room temperature using 1,8-diazabicyclo[5.4.0]undec-7-ene (DBU) as a catalyst for $1 \mathrm{~h}$. PS- $b$-PLA was obtained by precipitating in methanol after termination with benzoic acid. The final PS- $b$-PLA had a total Mn $=63 \mathrm{kDa}$, with a PLA volume fraction of 0.28 (by volume) yielding a cylindrical morphology with a polydispersity index of 1.08 , as determined with ${ }^{1} \mathrm{H}$ Nuclear Magnetic Resonance (NMR) and Size-Exclusion Chromatography (SEC) [34].

\subsection{Thin Film Preparation}

Typical solutions of $1.5 \%(\mathrm{w} / \mathrm{v})$ PS- $b$-PLA in toluene (non-selective solvent) were spin coated onto HMDS treated, natively-oxidized silicon wafers $(20 \mathrm{~mm} \times 20 \mathrm{~mm}$ ). HMDS treatment of the $\mathrm{Si}$ wafers was carried out by ultrasonically cleaning substrates in organic solvents (acetone followed by methanol), treating them in a 1:5 (v/v) HMDS:toluene solution for $16 \mathrm{~h}$, then rinsing in toluene to remove any excess HMDS that had not grafted fully to the substrate, and blowing dry with $\mathrm{N}_{2}$ gas. Treated wafers were then placed in a $75^{\circ} \mathrm{C}$ oven to remove any residual solvent. The films were spin coated at 1000-3000 rpm for $30 \mathrm{~s}$, diced into $12-16$ pieces $(\sim 5 \mathrm{~mm} \times 5 \mathrm{~mm})$, and immediately placed in a $75{ }^{\circ} \mathrm{C}$ (below the glass transition temperature of either block [34]) oven for drying. This process yielded $\sim 60 \mathrm{~nm}$ thick films, dependent on exact spin speed and solution concentration. Samples were dried for a minimum of one day. Periodic atomic force micrographs indicate no apparent aging issues or annealing effects while storing films at this temperature.

For the present experiment, thin films were hot-loaded into a $\mathrm{N}_{2}$ purged, over-pressurized solvent annealing chamber and immediately sealed to avoid water contamination. The samples were allowed to cool to room temperature and no further temperature control was implemented. This is described in full detail below. THF liquid was stored in a sealed Erlenmeyer flask over activated $3 \AA$ molecular sieves for several days before introducing it to the SAC, resulting in water levels of $4.1 \mathrm{ppm}$ or less [59]. Additional activated molecular sieves reside in the solvent reservoir of the SAC to continue active drying during subsequent solvent vapor anneals. Following solvent vapor exposure and complete solvent evaporation from the film, the samples were immediately transferred to a $0.05 \mathrm{M} \mathrm{NaOH}$ solution $\left(\mathrm{H}_{2} \mathrm{O}: \mathrm{CH}_{3} \mathrm{OH}=6: 4\right.$ by volume) for PLA minority domain degradation and left to soak for $45 \mathrm{~min}$, where the degradation rate is sensitive to molarity [60]. After removal, films were washed with deionized water/methanol for $5 \mathrm{~min}$. By removing the minority component immediately, this serves to ensure the morphology of the film is immobilized for subsequent imaging. Furthermore, to remove any additional surface contamination, a $150 \mathrm{~W}, 50 \mathrm{KHz} \mathrm{O}_{2}$ reactive ion etch (PE-50, PlasmaEtch, Inc., Carson, NV, USA) is employed for $10 \mathrm{~s}$ at 100 mTorr. This process removes $\sim 2-3 \mathrm{~nm}$ of organic material. Samples at this stage were immediately imaged with atomic force microscopy (AFM), without any further modification.

\subsection{Measuring Film Thickness}

Film thickness was determined with a Filmetrics F20-UV (San Diego, CA, USA) general-purpose film thickness measurement system with both halogen and deuterium sources. Spectral reflectance data was taken at differing time intervals (as discussed below), between $10 \mathrm{~ms}-3 \mathrm{~s}$ with a $10-250 \mathrm{~ms}$ integration time. Experimental data was modeled over a spectral range of $270-900 \mathrm{~nm}$ with a three-layer model ( $\mathrm{Si}+\mathrm{PS}-b$-PLA + air). We developed anticipated refractive index profiles based on known values (e.g., PS, $\mathrm{n}=1.59$; PLA, $\mathrm{n}=1.482 ; \mathrm{THF}, \mathrm{n}=1.407$ ). Therefore, we expected an index of refraction of 1.55 for the neat film, dropping to 1.45 with increasing solvent (THF) concentration up to $\phi=0.55$ [34]. Samples not following this trend in refractive index with increasing solvent were aborted and disposed. 


\subsection{Atomic Force Microscopy (AFM)}

Tapping mode AFM was performed on an Agilent 5420 microscope (Santa Clara, CA, USA) under ambient conditions using engagement setpoints between 0.9-0.95 of the free amplitude oscillation. The tapping mode cantilevers (BudgetSensors, Sofia, Bulgaria and Bruker, Billerica, MA, USA) had a resonant frequency of $300 \mathrm{kHz}$ and a force constant of $40 \mathrm{~N} / \mathrm{m}$. For imaging the film/substrate interface, the PLA-removed thin films were placed upside down on double-sided transparent tape (ScotchBrand, St. Paul, MN, USA) and placed in liquid $\mathrm{N}_{2}$ for $30 \mathrm{~s}$. Following liquid $\mathrm{N}_{2}$ exposure, the $\mathrm{Si}$ wafer was peeled away from the film providing access to the underside. These films were again exposed to a 10-20 s $\mathrm{O}_{2}$ reactive ion etch ( $150 \mathrm{~W} 50 \mathrm{KHz}$ in $100 \mathrm{mTorr}$ ) on the underside to remove any HMDS, a thin PS wetting layer or adhesive residue [34,61].

\section{Results}

\subsection{Design of a Purpose-Built Solvent Vapor Annealing Chamber}

The reproducibility of a final morphology and its propagation into solvent vapor-annealed BP films remains somewhat elusive, despite numerous advances in the field. To directly address this issue, we have designed, constructed and tested a purpose-built solvent vapor annealing chamber (SAC) that provides unparalleled control over introducing and maintaining precise solvent concentrations within the film during the annealing process as well as during the evaporation of solvent from the film. Concurrently, we maintain control over several additional necessary parameters (Table 1), including an exceptionally low dew point in the sample cell with active dry $\mathrm{N}_{2}$ purging, solvent vapor flow rate, film thickness as a proxy for solvent concentration in the film [34], chamber pressure, solvent selectivity, and substrate surface preparation. The present investigation uses $\sim 60 \mathrm{~nm}$ thick films of a prototypical BP, PS- $b$-PLA, that adopts a cylindrical morphology in the bulk. We use at room temperature a relatively neutral solvent, THF, for the anneal [29,34,62]; a PS-selective substrate, HMDS-functionalized $\mathrm{Si}$; and maintain a low dew point $\left(-100{ }^{\circ} \mathrm{C}\right)$ for the $\mathrm{N}_{2}$ carrier gas, due to the hygroscopic nature of THF and PLA. The following SAC description is a significant evolution over initial work in developing these protocols, including the addition of pneumatically-actuated, computer controlled solvent flow rates and valve actuation [34,38]. In our method, a copper-gasket sealed, all-metal chamber controls the SVA climate (i.e., humidity, chamber pressure, and evaporation times). The all-metal construction, except for chemically inert modified polytetrafluoroethylene sealed ball valves and a perfluoroelastomer sealed low-flow metering valve, ensures negligible interaction with the solvent during the annealing process. In particular, THF can be particularly aggressive on traditional organic gaskets and lubricants. Following hundreds of hours of exposure to THF, no degradation to any valves in the chamber is evident.

The chamber, shown in Figure 2 (additional images are available in Appendix A), is connected to an actively dried $\mathrm{N}_{2}$ line (dew point guaranteed to $-100^{\circ} \mathrm{C}$ ) (1), which splits into two $1 / 4^{\prime \prime}$ stainless steel tubing paths. Path 1 (in red) purges the sample space before annealing and during sample loading; this ensures a low dew point during the SVA. Additionally, BP films are hot-loaded from a $75{ }^{\circ} \mathrm{C}$ oven into the purged, over-pressurized chamber via a Conflat-flanged door and immediately sealed to avoid water contamination and allowed to cool to room temperature. Path 1 is also vital to the controlled evacuation of solvent vapor from the SAC during the evaporation phase. $\mathrm{N}_{2}$ flow is passed through an acrylic, block-style flowmeter (3-30 SCFH or 30-300 SCFH, dependent on flow rate chosen) to control and measure flow rates during solvent evaporation. 
Table 1. A compilation of variables considered in the design of solvent vapor annealing protocols, along with their importance and the steps taken in the design to address them.

\begin{tabular}{|c|c|c|}
\hline Variable & Importance & Protocols in Design \\
\hline Humidity & $\begin{array}{l}\text { Water is a polar solvent, which } \\
\text { will modify the solubility. } \\
\text { For example, water is } \\
\text { PLA selective }\end{array}$ & $\begin{array}{l}\text { Samples are stored in } 75^{\circ} \mathrm{C} \text { oven } \\
\text { Samples are hot loaded into chamber } \\
\text { Vacuum-grade, copper gaskets and } \\
\text { Swagelok seals throughout chamber } \\
\text { Molecular sieve-dried solvent } \\
\text { (tetrahydrofuran (THF)) } \\
\left.\text { Actively purged (dry } \mathrm{N}_{2}\right) \text { sample cell } \\
\text { Drierite Gas Purifier }\left(-100^{\circ} \mathrm{C} \text { dewpoint) }\right.\end{array}$ \\
\hline Solvent Vapor Flow Rate & $\begin{array}{l}\text { Flow rate is proportional to } \\
\text { solvent uptake in film }\end{array}$ & $\begin{array}{l}\text { Computer-controlled mass flow controller } \\
\text { Low-flow metering outlet valve }\end{array}$ \\
\hline Solvent Concentration & $\begin{array}{l}\text { Solvent concentration in film } \\
\text { during solvent vapor annealing } \\
\text { (SVA) modifies mobility }\end{array}$ & $\begin{array}{l}\text { In situ optical detection of solvent } \\
\text { concentration, assuming proportional to } \\
\text { film thickness, with } 10-20 \mathrm{~ms} \text { resolution }\end{array}$ \\
\hline Solvent Evaporation Rate & $\begin{array}{l}\text { Evaporation rate in linked to } \\
\text { morphology alignment }\end{array}$ & $\begin{array}{l}\text { Computer-controlled pneumatic valves } \\
\text { Variable flow } \mathrm{N}_{2} \text { purge line }\end{array}$ \\
\hline Initial Thickness & $\begin{array}{l}\text { Role of commensurate thickness } \\
\text { on morphology }\end{array}$ & $\begin{array}{l}\text { Films are all spun cast at a constant spin } \\
\text { speed from the same solution } \\
\text { concentration }\end{array}$ \\
\hline Vapor Pressure & $\begin{array}{l}\text { Vapor pressure during SVA } \\
\text { modifies solvent uptake and } \\
\text { evaporation }\end{array}$ & $\begin{array}{l}\text { High and low pressure gauges } \\
\text { Low-flow metering outlet valve can finely } \\
\text { adjust vapor pressure }\end{array}$ \\
\hline Solvent Selectivity & $\begin{array}{l}\text { Solvent selectivity modifies } \\
\text { surface energy and } \\
\text { polymer-polymer interactions }\end{array}$ & $\begin{array}{l}\text { THF is a relatively neutral solvent for PS } \\
\text { and PLA. It has slight PS selectivity }\end{array}$ \\
\hline Surface Selectivity & $\begin{array}{l}\text { Substrate preparation modifies } \\
\text { surface energy }\end{array}$ & $\begin{array}{l}\text { HMDS-functionalized Si substrate surface } \\
\text { promotes PS (majority block) adhesion }\end{array}$ \\
\hline
\end{tabular}

Path 2 (in green) flow is governed by a mass flow controller (2) connected to a LabVIEW-enabled computer (National Instruments, Austin, TX, USA). Flow rates range from 0-500 SCCM, dependent on intended anneal conditions. The flow of the metered, actively dried $\mathrm{N}_{2}$ gas is computer controlled via solenoid actuated pneumatic valves (3) that control $80 \mathrm{psi}(551 \mathrm{kPa}$ ) air flow to each of four pneumatic process valves (4). Following the flow controller, the $\mathrm{N}_{2}$ gas is passed through a primary safety valve (4) (this protects the mass flow controller from liquid solvent exposure). The controlled gas flow continues on its way to a sealed, molecular-sieve-dried solvent reservoir (5), a stainless steel Conflat tee with view window (Figure A5). This reservoir contains an additional inlet tube with a normally closed plug valve that is used for liquid solvent loading without breaking any Conflat seals Figure A6). The solvent reservoir is backed by a safety reservoir (6), a stainless steel Conflat tee with viewing window. The safety reservoir will collect fluid solvent if backpressure is present, avoiding exposure to the mass flow controller. The safety reservoir contains an additional tube with a normally closed plug valve to remove any liquid solvent without opening any Conflat seals. Ultimately, the dry $\mathrm{N}_{2}$ is bubbled through the solvent reservoir (5), which subsequently carries solvent in the vapor phase into the sample space (7), a 6-way Conflat cube $(70 \mathrm{~mm} \times 70 \mathrm{~mm} \times 70 \mathrm{~mm})$, via a computer-controlled, pneumatically-actuated chamber valve (4). All flow into the sample space exits through $3 / 4$ " stainless steel tubing via a pneumatically-actuated ball valve (8) or a low-flow metering valve (9) to a fume hood. The computer controlled, pneumatically-actuated valves (4), with compressed air inlet (10) and $120 \mathrm{~V}$ solenoid valves (3), allow us to quickly initiate and terminate the SVA with a specified level of timing. Of critical importance, initial testing indicates a controlled SVA evaporation time down to $15 \mathrm{~ms}$ or any time longer with 10-20 ms temporal resolution. To our knowledge, this is the fastest recorded SVA evaporation time for a BP thin film. 

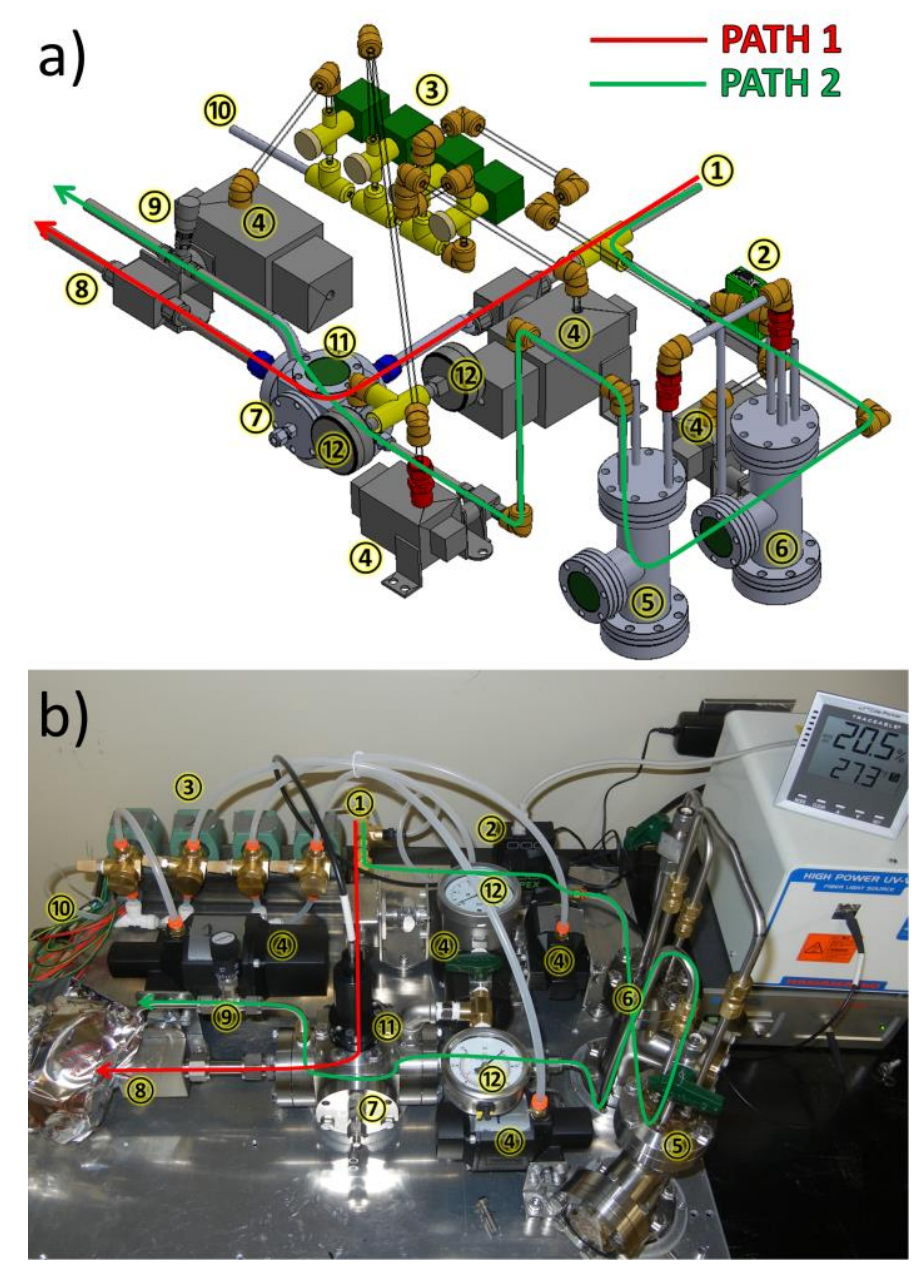

Figure 2. (a) Computer-aided design (CAD) and (b) real image of a computer-controlled, pneumatically actuated solvent vapor annealing chamber. More images can be found in Appendix A. Numbers and colored arrows are described in the text.

The BP sample resides in the sample space (7) on a custom-built mount (Figure A7). The sample mount features two recessed ports, which speeds sample loading and helps avoid direct flow from Paths 1 and 2 that can shift sample position. One recessed port holds the BP film, while the other holds a blank Si wafer for use as an optical standard. Recess ports are sized to hold up to an $8 \mathrm{~mm} \times 8 \mathrm{~mm} \mathrm{BP}$ film and a blank Si wafer. The chamber offers direct optical access to the sample space through a fused silica viewport ${ }^{11}$, enabling continuous monitoring of the sample chamber with high precision pressure gauges (12) to directly measure chamber pressure and in situ spectral reflectance-based measurements of film thickness with $0.1 \mathrm{~nm}$ thickness and 10-20 ms temporal resolution. These thickness measurements are directly related to solvent volume concentration $(\phi)$ within the film by:

$$
\phi=\frac{V_{\text {solvent }+ \text { film }}-V_{\text {film }}}{V_{\text {solvent }+ \text { film }}}=\frac{t_{\text {solvent }+ \text { film }}-t_{\text {film }}}{t_{\text {solvent }+ \text { film }}}=1-\frac{t_{\text {film }}}{t_{\text {solvent }+ \text { film }}}
$$

where $\phi$ is solvent concentration, $V$ denotes volume and $t$ is film thickness. As supported by direct observation, the areas for the two film states (i.e., swollen versus dry) are taken to be nominally identical and thus only a thickness measurement is required to obtain a real-time in situ probe of $\phi$ during SVA. 


\subsection{Theory of Operation}

Through the active control of solvent vapor inlet and outlet flows, the solvent concentration within the BP film is controlled and monitored as a function of time. As shown in Figure 3, using an in situ measurement of $\phi$, we divide the SVA process of a $60 \mathrm{~nm}$ PS- $b$-PLA film into four distinct time regimes. During the first three time regimes, thickness data was taken every $3 \mathrm{~s}$ with a $249 \mathrm{~ms}$ integration time. The initial regime (blue) includes the opening of the solvent reservoir to the sample chamber and an initial, constant $\mathrm{N}_{2}$ inlet flow of 30-100 SCCM (dependent on desired chamber pressure, 50 SCCM in the present study), resulting in an exponential increase in $\phi$. The thickness data is well modeled assuming a copolymer refractive index of 1.55 , consistent with the neat PS- $b$-PLA film (Appendix B). This exponential region typically lasts $\sim 60 \mathrm{~s}$ where the time dependence is well modeled with a single rate constant $\left(0.03-0.1 \mathrm{~s}^{-1}\right)$ with $0.088 \mathrm{~s}^{-1}$ for Figure 3 (see Figure A8 for the exponential fit), which is dependent on exact inlet and outlet flow rates. Outlet flow rates are governed by a low flow micrometer relief valve, which is set to a flow coefficient, $C_{v}$, of 0.0002-0.002 (1-6 turns), with 0.0004 being used in Figure 3. This, along with inlet flow, dictates the chamber pressure, which was maintained below $3.5 \mathrm{kPa}$ to obtain Figure 3. Following the displacement of residual $\mathrm{N}_{2}$ in the chamber with solvent vapor, the solvent uptake enters a second regime (red). This regime involves the metered uptake of solvent into the film, indicated by a controlled, linear increase in thickness over a period of $2 \mathrm{~min}$ (highly tunable, based on relative flow rates), until the film reaches the targeted $\phi$. The inlet and outlet flow rates remained the same for the second regime as given for the first regime in Figure 3, demonstrating this change in solvent uptake. The thickness data is well modeled assuming a copolymer refractive index that gradually approaches 1.45 , consistent with a volumetric combination of neat PS- $b$-PLA and THF (Appendix B). During this phase, two possible methods drive solvent into the film and increase the thickness. If the inlet flow is higher than outlet flow, the increasing pressure in the chamber will increasingly force solvent into the film (a relatively fast mechanism). If the inlet and outlet flows are comparable, the increase in relative solvent concentration within the chamber leads to an increased uptake of solvent into the film (a relatively slow mechanism). Therefore, it is possible to swell a film with a chamber pressure that is nominally atmospheric. Dependent on desired chamber pressure and ultimate thickness, the inlet and outlet flow rates are tuned. Precise control is best achieved by adjusting the mass flow controller-regulated inlet. We have not seen any impact with the rate of swelling in this second regime on the final morphology of the film, other than a potential dependence on the pressure in the chamber (faster swelling is typically accomplished with an increase in chamber pressure). The thickness data in this regime is well modeled assuming a copolymer refractive index of 1.45 (Figure A10), consistent with a solvated PS- $b$-PLA film containing $\phi=0.55$.

As the target $\phi$ is approached, decreasing the inlet flow or increasing the outlet flow causes $\phi$ to level off. The third regime (green) is characterized by an extremely constant (standard deviation is regularly less than $\Delta \phi=0.002$ ) solvent concentration. This constant concentration can be maintained for nearly any specified anneal time (3 min is shown in Figure 3, but we have maintained similar consistency for more than an hour). It is maintained through slight manual variations in inlet/outlet flow rates, with the future potential of computer feedback control. Figure 3 was achieved with a fixed outlet flow, consistent with regimes 1 and 2 and by controlling the inlet flow between 10-30 SCCM to achieve a constant thickness (solvent concentration). In the final seconds of this period, the integration time and thickness acquisition interval are switched to $10 \mathrm{~ms}$ and $0 \mathrm{~s}$, respectively. This is the fastest we can acquire spectral reflectance data and still get a high-fidelity model to extract thickness. This rapid data acquisition allows for close examination of the solvent evaporation period. In the fourth regime (magenta), evaporation of the solvent from the film occurs. Computer-controlled, pneumatically-actuated valves open Path 1 and close Path 2 . Through the release of pressure in the chamber, in tandem with $\mathrm{N}_{2}$ flow, the solvent vapor is released from the film and evacuated from the chamber. Details on timing control, which are dependent on Path 1 flow rates and chamber pressure, are given below. The initial evaporation of solvent from the film is not complete, where $\phi \approx 0.2$ 
typically remains in the film over the first $100 \mathrm{~ms}$. This remaining solvent typically takes an additional $1.5-10 \mathrm{~s}$ to be fully removed from the film, dependent on the Path 1 inlet flow rate. At $\phi<0.25-0.3$, the film is likely vitrified with exceptionally slow kinetics, consistent with recent GISAXS results [32]. Therefore, the final morphology is well locked-in during the initial, potentially fast, evaporation phase. In fact, atomic force micrographs of the free surface and the substrate surface (after removal from the Si substrate) of a PS- $b$-PLA film verify that a vertically-aligned cylindrical morphology can persist through an entire $60 \mathrm{~nm}$ film to the substrate surface (Figure 4 ). The PS- $b$-PLA film in Figure 4 was swollen with THF to $\phi=0.55$ and the solvent was subsequently evaporated out of the film (down to $\phi=0.2$, sufficient to vitrify the film) in $15 \mathrm{~ms}$. No condensation of solvent was observed during any changes in pressure for the present investigation.

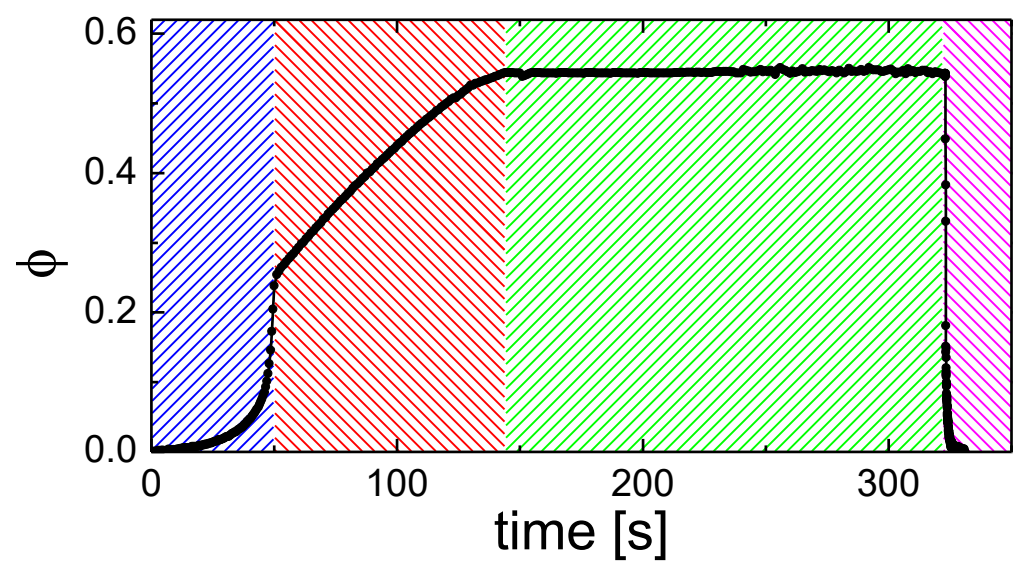

Figure 3. Solvent concentration in a BP film $(\phi)$ versus anneal time for a typical solvent vapor anneal of a $60 \mathrm{~nm}$ PS- $b$-PLA thin film. The blue indicates the initial solvent uptake regime. The red indicates the metered solvent uptake regime. The green indicates a fixed solvent concentration regime. The magenta indicates the solvent evaporation regime.

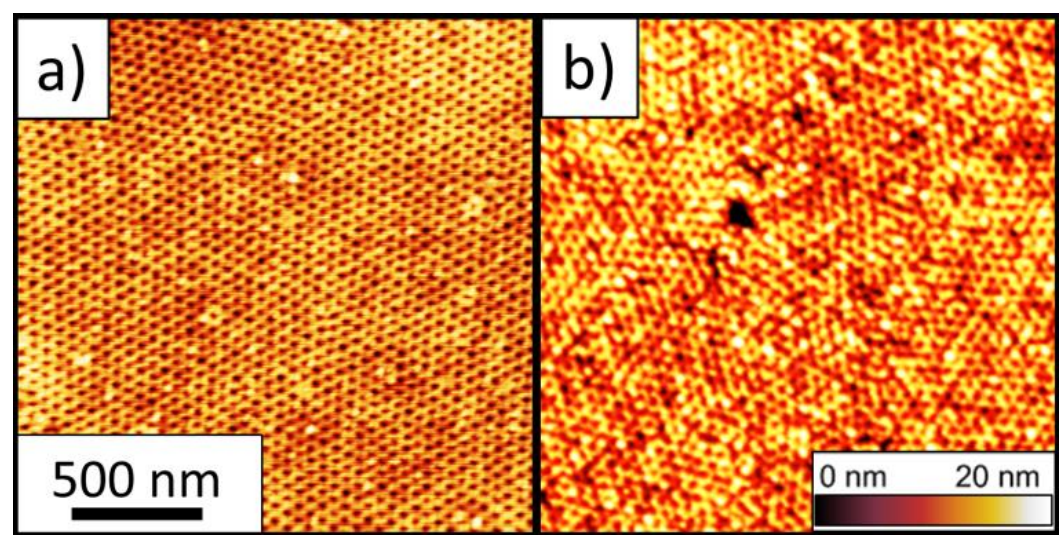

Figure 4. Atomic force topography micrographs of the free surface (a) and substrate surface (b) of a $60 \mathrm{~nm}$ PS- $b$-PLA film that was exposed to a THF concentration $\phi=0.55$ and having a solvent evaporation occurring in $15 \mathrm{~ms}$. Images were taken following hydrolytic removal of the PLA minority component and a brief $(10 \mathrm{~s}) \mathrm{O}_{2}$ reactive ion etch. The false color height scale is $20 \mathrm{~nm}$.

\subsection{Solvent Evaporation}

While a primary outcome of this purpose-built chamber is the ability to keep an arbitrary solvent concentration constant within a BP film for a specified time, another beneficial consequence is the ability to observe and control the solvent evaporation phase (fourth regime-solvent evaporation magenta 
regime-in Figure 3) with 10-20 ms temporal resolution. This high acquisition rate has revealed subtle differences in precise evaporation trajectories, dependent on exact chamber pressure and inlet/outlet flow rates. First, of importance to our specific chamber, is the timing between the inlet and outlet valves of Path 1. It is necessary to introduce a slight time delay between their actuation. This is detailed in Figure 5a. If the valves are open concurrently (i.e., $0 \mathrm{~ms}$ time delay), the finite impedance of the outlet tube causes a brief pressure spike in the chamber that drives residual solvent vapor from the chamber into the film. This increases the concentration within the film, possibly to disorder, ahead of the evaporation. This leads to inconsistent morphologies and is undesirable. If the outlet valve is opened far ahead of the inlet valve, a two-stage evaporation tends to take place. The opening of the outlet valve releases pressure from the chamber and leads to a drop in concentration within the film. Presumably, some solvent was retained in the film simply due to the finite pressure in the chamber during the anneal. Then, the opening of the inlet flow removes the remaining solvent in the chamber and the solvent is fully evaporated from the film. For example, a delay of $100 \mathrm{~ms}$ has two distinct evaporation trajectories, as seen in Figure 5a. For our specific system, a computer-controlled time delay of $25 \mathrm{~ms}$ leads to optimally fast evaporation without the associated pressure spike (Figure 5a).

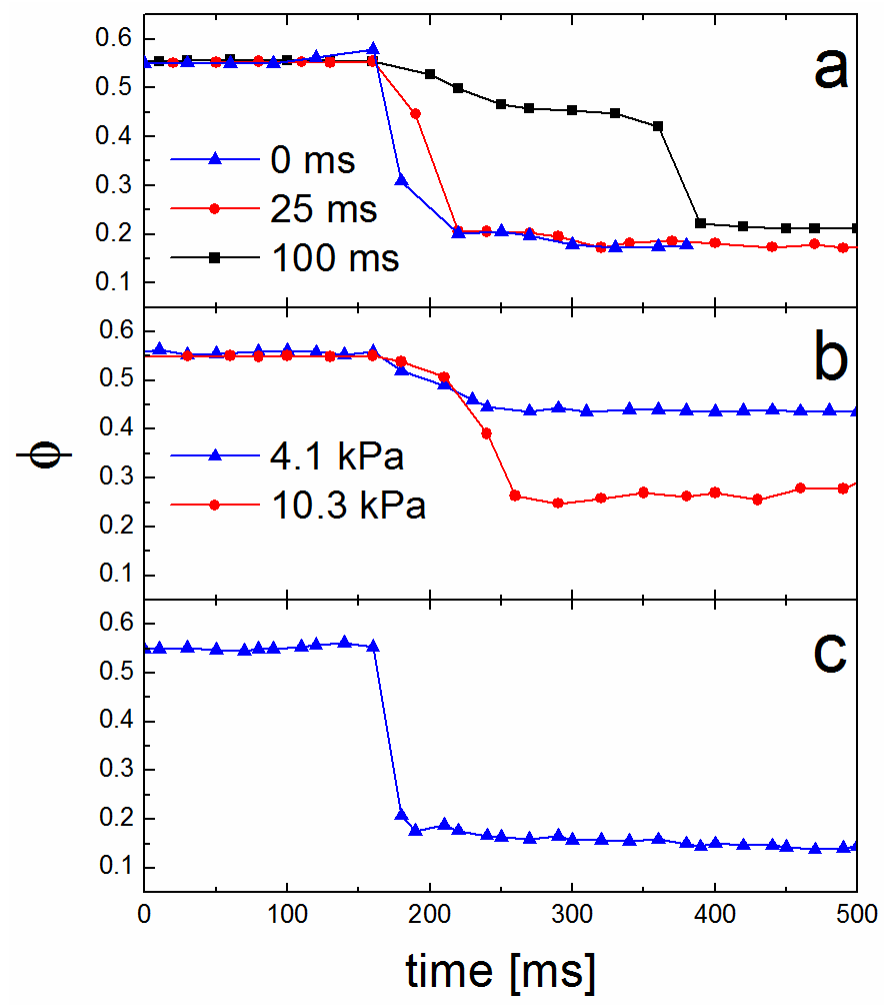

Figure 5. Solvent concentration $(\phi)$ versus anneal time during solvent evaporation; (a) solvent evaporation trajectories for three time delays between opening the chamber outlet to atmosphere and introducing an inlet flow of $\mathrm{N}_{2} ;(\mathbf{b})$ solvent evaporation trajectories for an annealing chamber with a relatively high or low vapor pressure; (c) Solvent evaporation trajectory for a fast evaporation with high inlet flow and zero vapor pressure in the chamber.

Second, considering the role of chamber pressure during the evaporation phase; the release of pressure within the chamber as the outlet valve of Path 1 is opened leads to a release of solvent from the film. This is true even in the complete absence of any inlet $\mathrm{N}_{2}$ flow, as shown in Figure $5 \mathrm{~b}$. As expected, the higher the pressure in the chamber, the more dramatic the decrease in solvent concentration in the film when the outlet valve is opened. In the case where pressure in the chamber approaches $10.3 \mathrm{kPa}$, the pressure drop to $0 \mathrm{kPa}$ is sufficient to remove solvent down to $\phi=0.25$. This concentration is sufficiently low to lock-in the morphology where only kinetically slow vitrification persists, discussed 
above. In the case where chamber pressure is somewhat lower, $4.1 \mathrm{kPa}$, the release of pressure to $0 \mathrm{kPa}$ is insufficient to remove a sufficient amount of solvent to vitrify the film without additional inlet $\mathrm{N}_{2}$ flow. Finally, considering inlet and outlet flows (Figure 5c), a higher Path 1 inlet flow rate leads to optimally fast solvent vapor removal from the chamber. This potentially leads to rapid solvent evaporation from the film (10-20 ms). This rapid evaporation causes a strong ordering front and tends to drive cylinder propagation perpendicular to the film (Figure 4). After reviewing 700 trials following these SVA protocols, including rapid evaporation $(\sim 15 \mathrm{~ms})$ and low chamber pressure, $640(91.4 \%)$ films showed full perpendicular alignment, 14 (2.0\%) showed full in-plane alignment, and 46 (6.6\%) exhibited a mixed, mostly perpendicular alignment with some in-plane cylinders evident. The latter two cases (in-plane cylinders or mixed) were regularly attributed to elevated ambient humidity in the lab. Clearly, the control over both chamber pressure and Path 1 flows provide extensive control over the solvent removal rate and its specific trajectory. The resultant morphologies and their propagation into the bulk of a film for these different trajectories are the subject of a forthcoming manuscript.

\section{Conclusions}

It is increasingly clear that annealing BP thin films is critical to achieving self-assembled nanostructures with a given morphology and lateral order. Solvent-based techniques have proven to be highly effective due to the dramatically increased polymer chain mobility while mitigating thermal degradation. In particular, chain mobility near the order disorder transition is optimally enhanced. In addition, the evaporation of solvent from a film is critical in determining the propagation of a given morphology into the bulk of the film. At present, there are three primary methods for incorporating solvent into a BP film; however, each is limited in its ability to directly control solvent concentration within the film and the solvent evaporation rate. These limitations have stifled investigation into the time-dependence of these effects. Furthermore, solvent-enhanced crystallization has evolved from BPs to other organic systems, with conjugated polymer/organic photovoltaics being one illustrative example [63], further indicating its potential efficacy. Therefore, we have presented a purpose-built solvent vapor annealing chamber that was designed and constructed to elucidate the role of key parameters involved in directed self-assembly in BP thin films with goals of enhanced reliability, repeatability and the eventual scaling up of the SVA process. Currently, the use of in situ scattering techniques, such as GISAXS or GISANS, are critical for understanding the ordering mechanisms of block polymer films. However, they may prove impractical for industrial applications. Therefore, transferring protocols developed with those techniques to purpose-built chambers, such as the one presented here, could be essential for proliferation. In particular, there is interest in observing and controlling the mechanisms for increased correlation lengths of self-assembled features and the growth propagation of those features into the bulk of the film in the final film state (i.e., following solvent evaporation). This level of control opens up possibilities for a variety of morphology controls. Moreover, in contrast to many current efforts, the technique does not involve extensive in situ monitoring with advanced scattering techniques, but could serve to enhance those techniques with higher temporal resolution and control. Rather, the present in situ monitoring relies on inexpensive, readily available optical techniques in conjunction with pneumatically-actuated, computer-controlled flow controllers and valves. Such low-cost and simple methods could prove useful for any future scaling-up of this process. 
Author Contributions: Conceived and designed-G.N., R.G. and A.B. Performed the experiments-G.N., C.S.D., M.A.G., J.W. Analyzed the data-G.N., C.S.D., M.A.G., J.W. and A.B. Wrote the paper-G.N. and A.G.B. Supervised work-A.B. All authors discussed the results and implications and commented on the manuscript at all stages.

Acknowledgments: This work was supported by the National Science Foundation through the Nebraska EPSCoR FIRST Award under contract 1004094. Additional support came from the Omaha Public Power District and Creighton University's Center for Undergraduate Research and Scholarship. G.N. and R.G. acknowledge support from the NASA Nebraska Space Grant Consortium. C.S.D. acknowledges support from the Clare Boothe Luce foundation. We acknowledge mechanical expertise and insight from Brad Walters and Rustin Haase.

Conflicts of Interest: The authors declare no conflict of interest.

\section{Appendix A}

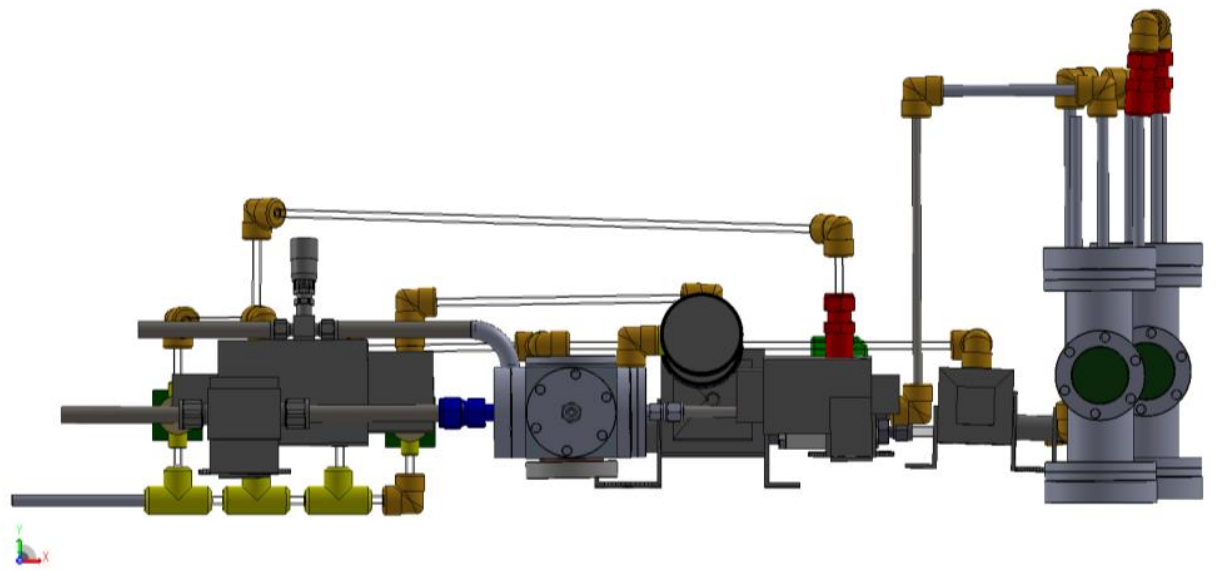

Figure A1. Front view of the solvent vapor annealing (SVA) chamber.

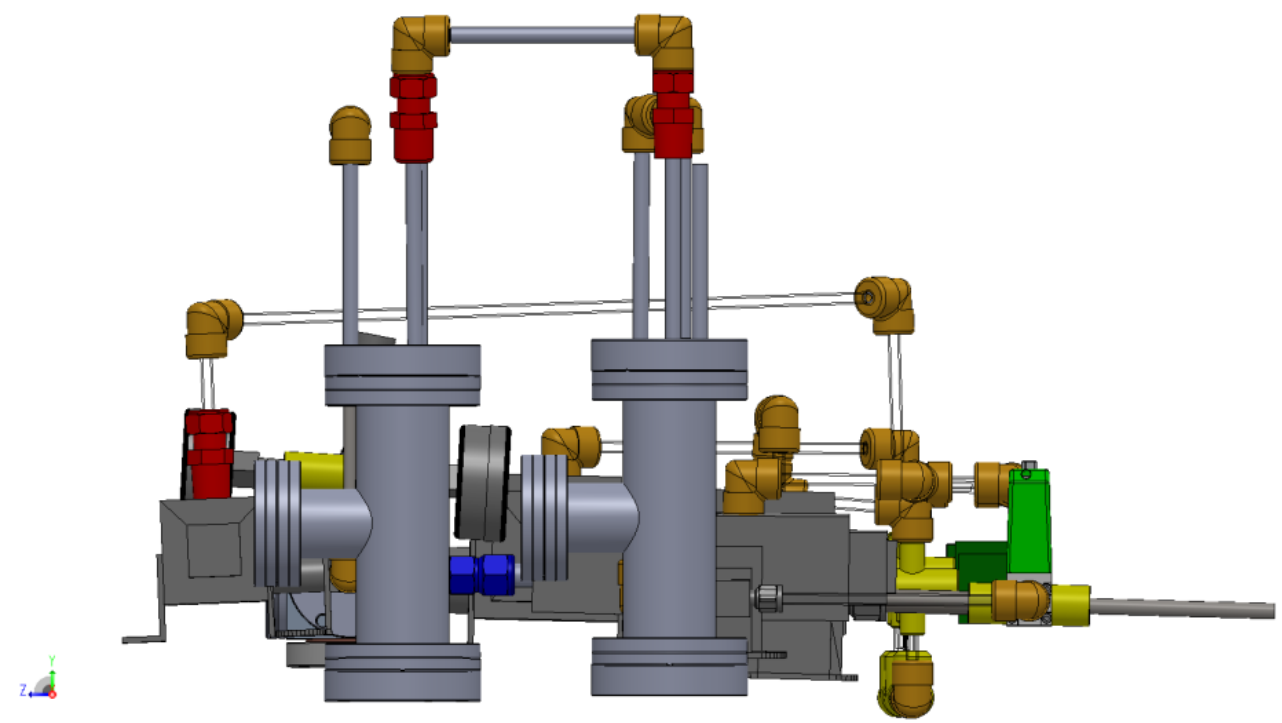

Figure A2. Right view of the SVA chamber. 


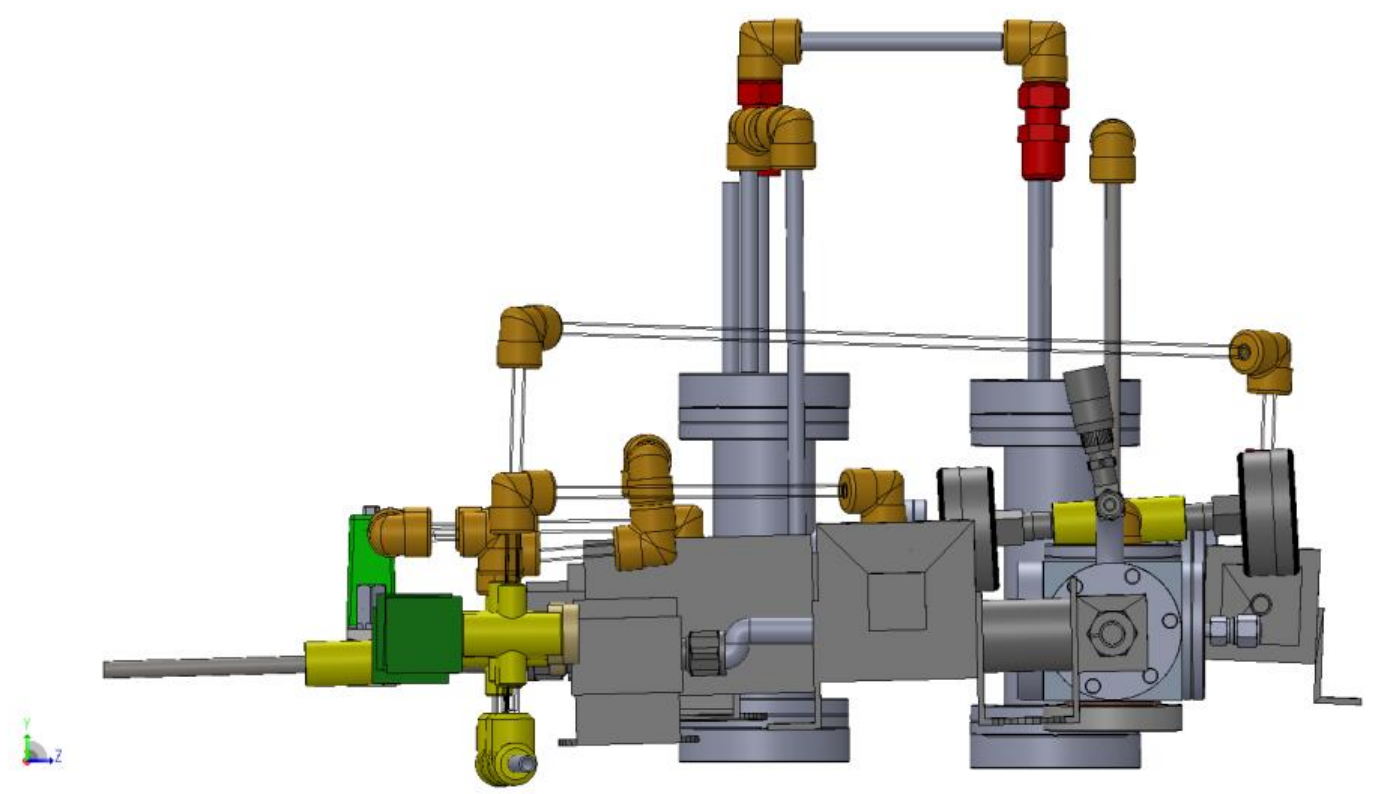

Figure A3. Left view of the SVA chamber.

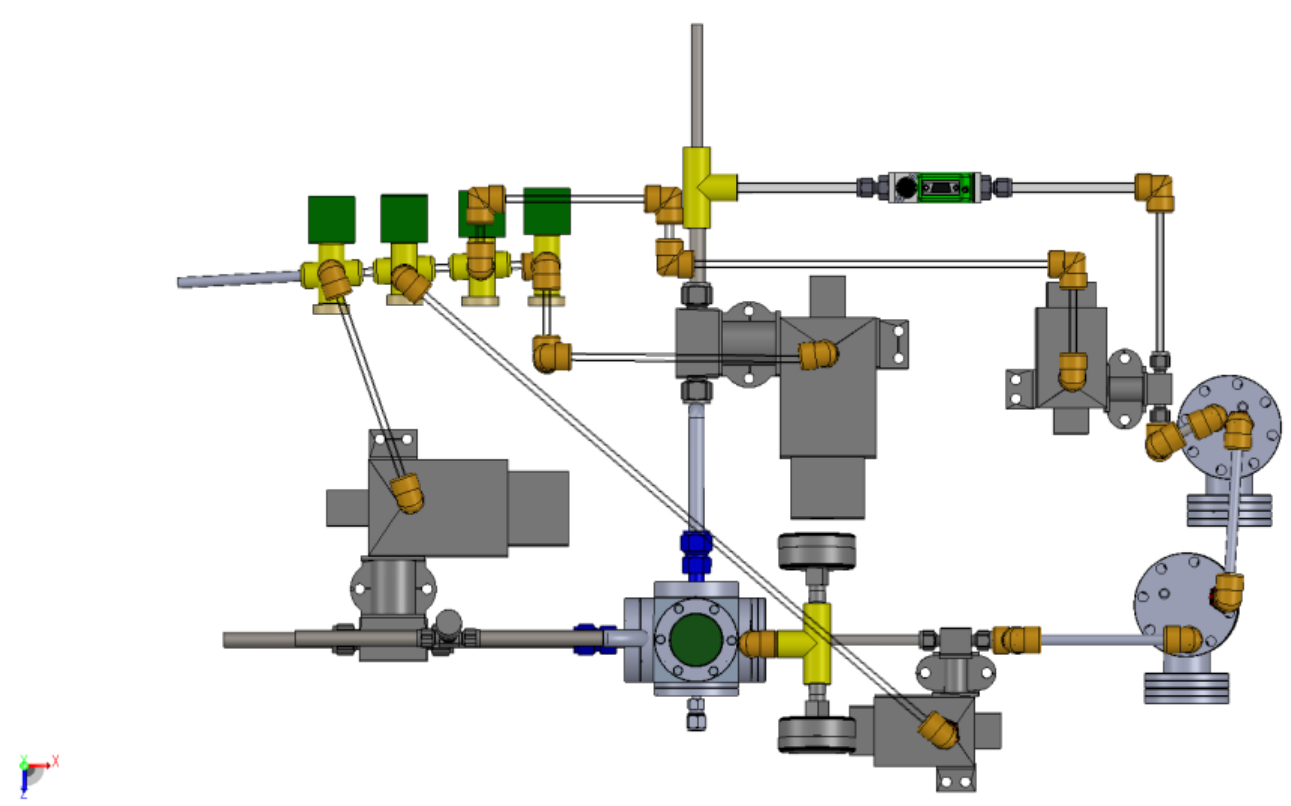

Figure A4. Top view of the SVA chamber. 


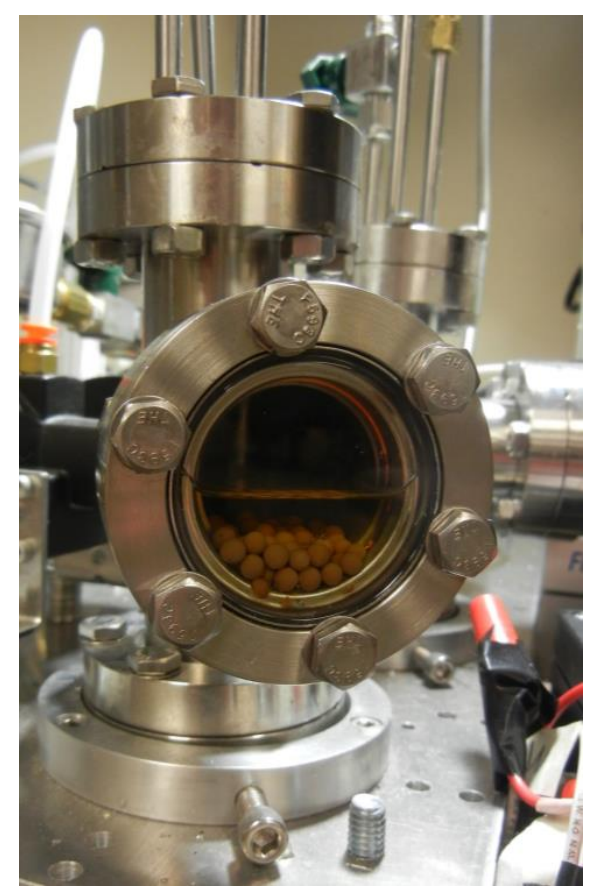

Figure A5. Solvent reservoir with optical view port, liquid solvent, and molecular sieves.

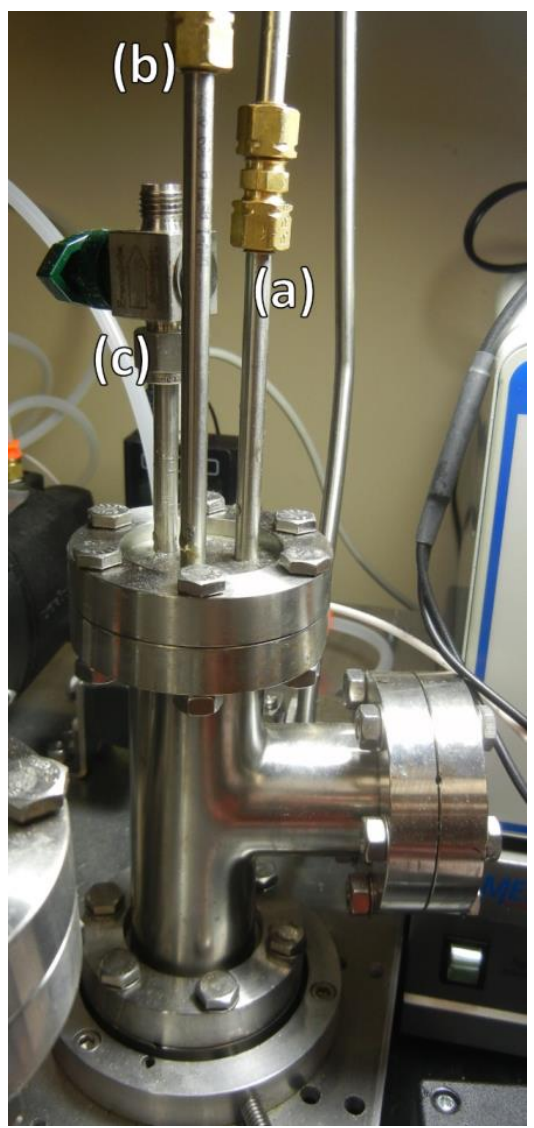

Figure A6. Depiction of the solvent reservoir with three $\frac{1}{4}$ " tubing ports. One tube (a) is an $\mathrm{N}_{2}$ inlet that extends to the bottom of the reservoir. One tube $(\mathbf{b})$ is a solvent vapor outlet that only extends into the reservoir $\frac{1}{2}$ " to capture the vapor. One tube (c) is capped with a normally-closed plug valve. This is used to add liquid solvent without opening the Conflat flanges. 


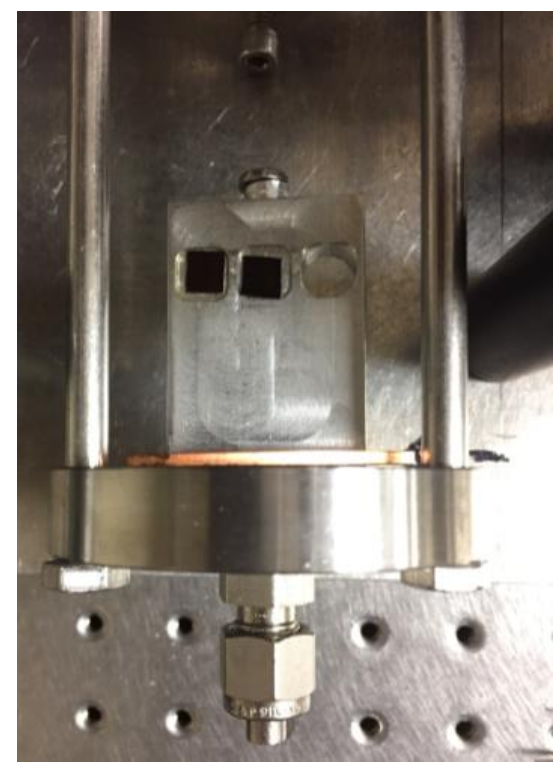

Figure A7. Custom designed sample holder. The block copolymer film sits in the center well, a silicon optical standard sits in the left well. A hole for background ultraviolet-visible (UV-V) light is spectroscopy subtraction is on the right. The sample holder rests on a two-pronged frame to slide the samples in and out of the main chamber and is sealed with a Conflat flange with copper gasket.

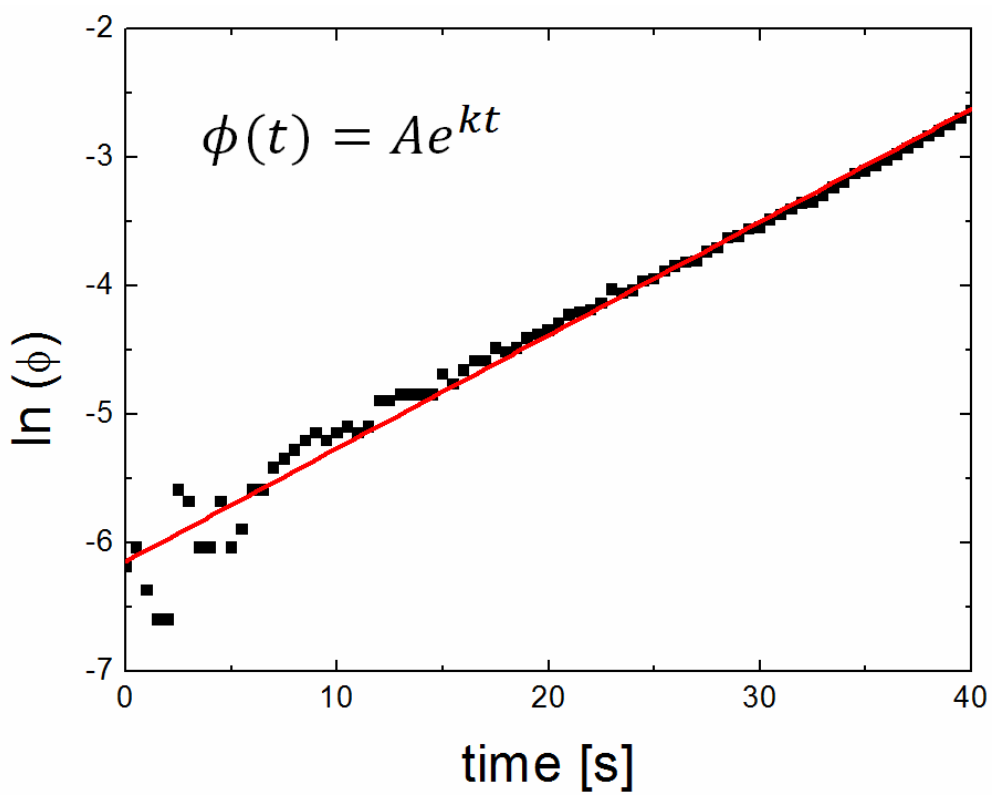

Figure A8. Best linear fit to the natural $\log$ of solvent concentration for the first $40 \mathrm{~s}$ (regime 1) of Figure 3, showing a single exponential rate constant of $0.088 \pm 0.0013 \mathrm{~s}^{-1}$. The $\mathrm{R}^{2}$ value of the fit is 0.982 . 


\section{Appendix B}

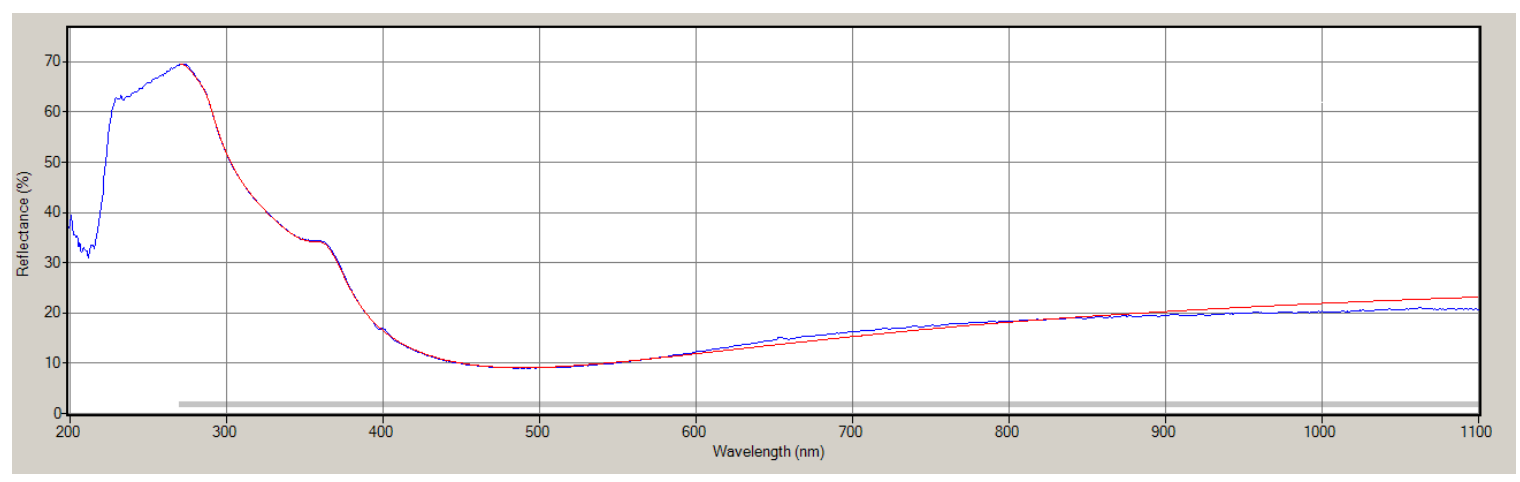

Figure A9. Spectral reflectance of a neat $60 \mathrm{~nm}$ polystyrene-block-polylactide film on silicon. The blue line is reflectance data; red line is the spectral reflectance model using an index of refraction of 1.55 for the copolymer layer.

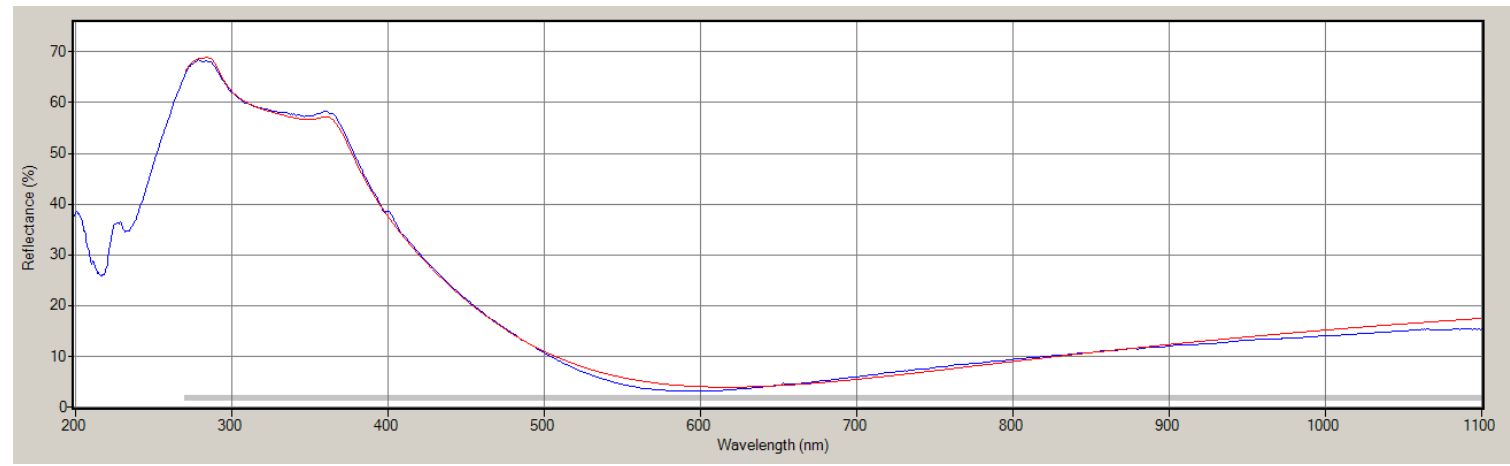

Figure A10. Spectral reflectance of a THF swollen polystyrene-block-polylactide film on Silicon. The film contains $55 \%$ THF solvent. The blue line is reflectance data; red line is the spectral reflectance model using an index of refraction of 1.45 for the copolymer layer.

\section{References}

1. Terris, B.D.; Thomson, T. Nanofabricated and self-assembled magnetic structures as data storage media. J. Phys. D Appl. Phys. 2005, 38, R199-R222. [CrossRef]

2. Borah, D.; Shaw, M.T.; Rasappa, S.; Farrell, R.A.; O’Mahony, C.; Faulkner, C.M.; Bosea, M.; Gleeson, P.; Holmes, J.D.; Morris, M.A. Plasma etch technologies for the development of ultra-small feature size transistor devices. J. Phys. D Appl. Phys. 2011, 44, 174012. [CrossRef]

3. Luo, Y.; Montarnal, D.; Kim, S.; Shi, W.; Barteau, K.P.; Pester, C.W.; Hustad, P.D.; Christianson, M.D.; Fredrickson, G.H.; Kramer, E.J.; et al. Poly(dimethylsiloxane- $b$-methyl methacrylate): A Promising Candidate for Sub-10 nm Patterning. Macromolecules 2015, 48, 3422-3430. [CrossRef]

4. Bang, J.; Jeong, U.; Ryu, D. Block copolymer nanolithography: Translation of molecular level control to nanoscale patterns. Adv. Mater. 2009, 21, 4769-4792. [CrossRef] [PubMed]

5. Bates, C.M.; Maher, M.J.; Janes, D.W.; Ellison, C.J.; Willson, C.G. Block copolymer lithography. Macromolecules 2014, 47, 2-12. [CrossRef]

6. Hamley, I.W. Ordering in thin films of block copolymers: Fundamentals to potential applications. Prog. Polym. Sci. 2009, 34, 1161-1210. [CrossRef]

7. Black, C.T.; Ruiz, R.; Breyta, G.; Cheng, J.Y.; Colburn, M.E.; Guarini, K.W.; Kim, H.-C.; Zhang, Y. Polymer self assembly in semiconductor microelectronics. IBM J. Res. Dev. 2007, 51, 605-633. [CrossRef]

8. Kim, H.-C.; Park, S.-M.; Hinsberg, W.D. Block copolymer based nanostructures: Materials, processes, and applications to electronics. Chem. Rev. 2010, 110, 146-177. [CrossRef] [PubMed] 
9. Stoykovich, M.P.; Nealey, P.F. Block copolymers and conventional lithography. Mater. Today 2006, 9, $20-29$. [CrossRef]

10. Guarini, K.W.; Black, C.T.; Yeung, S.H.I. Optimization of diblock copolymer thin film self assembly. Adv. Mater. 2002, 14, 1290-1294. [CrossRef]

11. International Technology Roadmap for Semiconductors (ITRS). 2013. Available online: https://www. semiconductors.org/main/2013_international_technology_roadmap_for_semiconductors_itrs/ (accessed on 18 April 2018).

12. Hamley, I.W. The Physics of Block Copolymers; Oxford University Press: Oxford, UK, 1998; ISBN 0198502184.

13. Bates, F.S. Polymer-polymer phase behavior. Science 1991, 251, 898-905. [CrossRef] [PubMed]

14. Anastasiadis, S.H.; Russell, T.P.; Satija, S.K.; Majkrzak, C.F. Neutron reflectivity studies of the surface-induced ordering of diblock copolymer films. Phys. Rev. Lett. 1989, 62, 1852-1855. [CrossRef] [PubMed]

15. Menelle, A.; Russell, T.P.; Anastasiadis, S.H.; Satija, S.K.; Majkrzak, C.F. Ordering of thin diblock copolymer films. Phys. Rev. Lett. 1992, 68, 67-70. [CrossRef] [PubMed]

16. Fasolka, M.J.M.; Mayes, A.M.A. Block copolymer thin films: Physics and applications. Annu. Rev. Mater. Res. 2003, 31, 323-355. [CrossRef]

17. Albert, J.N.L.; Epps, T.H., III. Self-assembly of block copolymer thin films. Mater. Today 2010, 13, $24-33$. [CrossRef]

18. Olayo-Valles, R.; Lund, M.S.; Leighton, C.; Hillmyer, M.A. Large area nanolithographic templates by selective etching of chemically stained block copolymer thin films. J. Mater. Chem. 2004, 14, 2729-2731. [CrossRef]

19. Zhang, X.; Harris, K.D.; Wu, N.L.Y.; Murphy, J.N.; Buriak, J.M. Fast assembly of ordered block copolymer nanostructures through microwave annealing. ACS Nano 2010, 4, 7021-7029. [CrossRef] [PubMed]

20. Morkved, T.L.; Lu, M.; Urbas, A.M.; Ehrichs, E.E.; Jaeger, H.M.; Mansky, P.; Russell, T.P. Local control of microdomain orientation in diblock copolymer thin films with electric fields. Science 1996, 273, 931-933. [CrossRef] [PubMed]

21. Thurn-Albrecht, T. Ultrahigh-density nanowire arrays grown in self-assembled diblock copolymer templates. Science 2000, 290, 2126-2129. [CrossRef] [PubMed]

22. Kubo, T.; Wang, R.F.; Olson, D.A.; Rodwogin, M.; Hillmyer, M.A.; Leighton, C. Spontaneous alignment of self-assembled ABC triblock terpolymers for large-area nanolithography. Appl. Phys. Lett. 2008, 93, 133112. [CrossRef]

23. Kubo, T.; Parker, J.S.; Hillmyer, M.A.; Leighton, C. Characterization of pattern transfer in the fabrication of magnetic nanostructure arrays by block copolymer lithography. Appl. Phys. Lett. 2007, 90, 233113. [CrossRef]

24. Ruiz, R.; Kang, H.; Detcheverry, F.A.; Dobisz, E.; Kercher, D.S.; Albrecht, T.R.; de Pablo, J.J.; Nealey, P.F. Density multiplication and improved lithography by directed block copolymer assembly. Science 2008, 321, 936-939. [CrossRef] [PubMed]

25. Sinturel, C.; Vayer, M.; Morris, M.; Hillmyer, M.A. Solvent vapor annealing of block polymer thin films. Macromolecules 2013, 46, 5399-5415. [CrossRef]

26. Kelly, J.Y.; Albert, J.N.L.; Howarter, J.A.; Kang, S.; Stafford, C.M.; Epps, T.H.; Fasolka, M.J. Investigation of thermally responsive block copolymer thin film morphologies using gradients. ACS Appl. Mater. Interfaces 2010, 2, 3241-3248. [CrossRef] [PubMed]

27. Di, Z.; Posselt, D.; Smilgies, D.-M.; Papadakis, C.M. Structural rearrangements in a lamellar diblock copolymer thin film during treatment with saturated solvent vapor. Macromolecules 2010, 43, 418-427. [CrossRef] [PubMed]

28. Sun, Y.; Henderson, K.J.; Jiang, Z.; Strzalka, J.W.; Wang, J.; Shull, K.R. Effects of reactive annealing on the structure of poly(methacrylic acid)-poly(methyl methacrylate) diblock copolymer thin films. Macromolecules 2011, 44, 6525-6531. [CrossRef]

29. Sinturel, C.; Grosso, D.; Boudot, M.; Amenitsch, H.; Hillmyer, M.A.; Pineau, A.; Vayer, M. Structural transitions in asymmetric poly(styrene)-block-poly(lactide) thin films induced by solvent vapor exposure. ACS Appl. Mater. Interfaces 2014, 6, 12146-12152. [CrossRef] [PubMed]

30. Gu, X.; Gunkel, I.; Hexemer, A.; Gu, W.; Russell, T.P. An in situ grazing incidence X-ray scattering study of block copolymer thin films during solvent vapor annealing. Adv. Mater. 2014, 26, 273-281. [CrossRef] [PubMed] 
31. Li, W.; Nealey, P.F.; de Pablo, J.J.; Müller, M. Defect removal in the course of directed self-assembly is facilitated in the vicinity of the order-disorder transition. Phys. Rev. Lett. 2014, 113, 168301. [CrossRef] [PubMed]

32. Gu, X.; Gunkel, I.; Hexemer, A.; Russell, T.P. Controlling domain spacing and grain size in cylindrical block copolymer thin films by means of thermal and solvent vapor annealing. Macromolecules 2016, 49, 3373-3381. [CrossRef]

33. Lodge, T.P.; Pudil, B.; Hanley, K.J. The full phase behavior for block copolymers in solvents of varying selectivity. Macromolecules 2002, 35, 4707-4717. [CrossRef]

34. Baruth, A.; Seo, M.; Lin, C.H.; Walster, K.; Shankar, A.; Hillmyer, M.A.; Leighton, C. Optimization of long-range order in solvent vapor annealed poly(styrene)-block-poly(lactide) thin films for nanolithography. ACS Appl. Mater. Interfaces 2014, 16, 13770-13781. [CrossRef] [PubMed]

35. Lin, C.-H.; Polisetty, S.; O’Brien, L.; Baruth, A.; Hillmyer, M.A.; Leighton, C.; Gladfelter, W.L.W.L. Size-tuned $\mathrm{ZnO}$ nanocrucible arrays for magnetic nanodot synthesis via atomic layer deposition-assisted block polymer lithography. ACS Nano 2015, 9, 1379-1387. [CrossRef] [PubMed]

36. Albert, J.N.L.; Bogart, T.D.; Lewis, R.L.; Beers, K.L.; Fasolka, M.J.; Hutchison, J.B.; Vogt, B.D.; Epps, T.H. Gradient solvent vapor annealing of block copolymer thin films using a microfluidic mixing device. Nano Lett. 2011, 11, 1351-1357. [CrossRef] [PubMed]

37. Luo, M.; Epps, T.H., III. Directed block copolymer thin film self-assembly: Emerging trends in nanopattern fabrication. Macromolecules 2013, 46, 7567-7579. [CrossRef]

38. Baruth, A.; Rodwogin, M.D.; Shankar, A.; Erickson, M.J.; Hillmyer, M.A.; Leighton, C. Non-lift-off block copolymer lithography of $25 \mathrm{~nm}$ magnetic nanodot arrays. ACS Appl. Mater. Interfaces 2011, 3, 3472-3481. [CrossRef] [PubMed]

39. Berezkin, A.V.; Papadakis, C.M.; Potemkin, I.I. Vertical domain orientation in cylinder-forming diblock copolymer films upon solvent vapor annealing. Macromolecules 2016, 49, 415-424. [CrossRef]

40. Berezkin, A.V.; Jung, F.; Posselt, D.; Smilgies, D.M.; Papadakis, C.M. Vertical vs. lateral macrophase separation in thin films of block copolymer mixtures: Computer simulations and GISAXS experiments. ACS Appl. Mater. Interfaces 2017, 9, 31291-31301. [CrossRef] [PubMed]

41. Berezkin, A.V.; Jung, F.; Posselt, D.; Smilgies, D.-M.; Papadakis, C.M. In situ tracking of composition and morphology of a diblock copolymer film with GISAXS during exchange of solvent vapors at elevated temperatures. Adv. Funct. Mater. 2018, 28, 1706226. [CrossRef]

42. Bang, J.; Kim, B.J.; Stein, G.E.; Russell, T.P.; Li, X.; Wang, J.; Kramer, E.J.; Hawker, C.J. Effect of humidity on the ordering of PEO-based copolymer thin films. Macromolecules 2007, 40, 7019-7025. [CrossRef]

43. Knoll, A.; Magerle, R.; Krausch, G. Phase behavior in thin films of cylinder-forming ABA block copolymers: Experiments. J. Chem. Phys. 2004, 120, 1105-1116. [CrossRef] [PubMed]

44. Zettl, U.; Knoll, A.; Tsarkova, L. Effect of confinement on the mesoscale and macroscopic swelling of thin block copolymer films. Langmuir 2010, 26, 6610-6617. [CrossRef] [PubMed]

45. Dehmel, R.; Dolan, J.A.; Gu, Y.; Wiesner, U.; Wilkinson, T.D.; Baumberg, J.J.; Steiner, U.; Wilts, B.D.; Gunkel, I. Optical imaging of large gyroid grains in block copolymer templates by confined crystallization. Macromolecules 2017, 50, 6255-6262. [CrossRef] [PubMed]

46. Gotrik, K.W.; Hannon, A.F.; Son, J.G.; Keller, B.; Alexander-Katz, A.; Ross, C.A. Morphology control in block copolymer films using mixed solvent vapors. ACS Nano 2012, 6, 8052-8059. [CrossRef] [PubMed]

47. Posselt, D.; Zhang, J.; Smilgies, D.-M.; Berezkin, A.V.; Potemkin, I.I.; Papadakis, C.M. Restructuring in block copolymer thin films: In situ GISAXS investigations during solvent vapor annealing. Prog. Polym. Sci. 2017, 66, 80-115. [CrossRef]

48. Jung, Y.S.; Ross, C.A. Solvent vapor induced tunability of self assembled block copolymer patterns. Adv. Mater. 2009, 21, 2540-2545. [CrossRef]

49. Bai, W.; Hannon, A.F.; Gotrik, K.W.; Choi, H.K.; Aissou, K.; Liontos, G.; Ntetsikas, K.; Alexander-katz, A.; Ross, C.A. Thin film morphologies of bulk-gyroid polystyrene-block- polydimethylsiloxane under solvent vapor annealing. Macromolecules 2014, 47, 6000-6008. [CrossRef]

50. Di, Z.; Posselt, D.; Smilgies, D.-M.; Li, R.; Rauscher, M.; Potemkin, I.I.; Papadakis, C.M. Stepwise swelling of a thin film of lamellae-forming poly(styrene-b-butadiene) in cyclohexane vapor. Macromolecules 2012, 45, 5185-5195. [CrossRef] 
51. Son, J.G.; Gotrik, K.W.; Ross, C.A. High-aspect-ratio perpendicular orientation of PS-b-PDMS thin films under solvent annealing. ACS Macro Lett. 2012, 1, 1279-1284. [CrossRef]

52. Albert, J.N.L.; Young, W.-S.; Lewis, R.L.; Bogart, T.D.; Smith, J.R.; Epps, T.H. Systematic study on the effect of solvent removal rate on the morphology of solvent vapor annealed ABA triblock copolymer thin films. ACS Nano 2012, 6, 459-466. [CrossRef] [PubMed]

53. Bai, W.; Yager, K.G.; Ross, C.A. In Situ Characterization of the Self-Assembly of a Polystyrenepolydimethylsiloxane block copolymer during solvent vapor annealing. Macromolecules 2015, 48, 8574-8584. [CrossRef]

54. Müller-Buschbaum, P. GISAXS and GISANS as metrology technique for understanding the 3D morphology of block copolymer thin films. Eur. Polym. J. 2016. [CrossRef]

55. Kim, S.H.; Misner, M.J.; Xu, T.; Kimura, M.; Russell, T.P. Highly oriented and ordered arrays from block copolymers via solvent evaporation. Adv. Mater. 2004, 16, 226-231. [CrossRef]

56. Nandan, B.; Vyas, M.K.; Böhme, M.; Stamm, M. Composition-dependent morphological transitions and pathways in switching of fine structure in thin films of block copolymer supramolecular assemblies. Macromolecules 2010, 43, 2463-2473. [CrossRef]

57. Paradiso, S.P.; Delaney, K.T.; García-Cervera, C.J.; Ceniceros, H.D.; Fredrickson, G.H. Block copolymer self assembly during rapid solvent evaporation: Insights into cylinder growth and stability. ACS Macro Lett. 2014, 3, 16-20. [CrossRef]

58. Phillip, W.; Hillmyer, M.; Cussler, E. Cylinder orientation mechanism in block copolymer thin films upon solvent evaporation. Macromolecules 2010, 43, 7763-7770. [CrossRef]

59. Williams, D.B.G.; Lawton, M. Drying of organic solvents: Quantitative evaluation of the efficiency of several desiccants. J. Org. Chem. 2010, 75, 8351-8354. [CrossRef] [PubMed]

60. Cummins, C.; Mokarian-Tabari, P.; Holmes, J.D.; Morris, M.A. Selective etching of polylactic acid in poly(styrene)-block-poly(D,L)lactide diblock copolymer for nanoscale patterning. J. Appl. Polym. Sci. 2014, 131, 40798. [CrossRef]

61. Zalusky, A.S.; Olayo-Valles, R.; Wolf, J.H.; Hillmyer, M.A. Ordered nanoporous polymers from polystyrene-polylactide block copolymers. J. Am. Chem. Soc. 2002, 124, 12761-12773. [CrossRef] [PubMed]

62. Vayer, M.; Hillmyer, M.A.; Dirany, M.; Thevenin, G.; Erre, R.; Sinturel, C. Perpendicular orientation of cylindrical domains upon solvent annealing thin films of polystyrene-b-polylactide. Thin Solid Films 2010, 518, 3710-3715. [CrossRef]

63. Miller, S.; Fanchini, G.; Lin, Y.-Y.; Li, C.; Chen, C.-W.; Su, W.-F.; Chhowalla, M. Investigation of nanoscale morphological changes in organic photovoltaics during solvent vapor annealing. J. Mater. Chem. 2008, 18, 306-312. [CrossRef]

(C) 2018 by the authors. Licensee MDPI, Basel, Switzerland. This article is an open access article distributed under the terms and conditions of the Creative Commons Attribution (CC BY) license (http://creativecommons.org/licenses/by/4.0/). 